Commun. Fac. Sci. Univ. Ank. Ser. A1 Math. Stat.

Volume 68, Number 2, Pages 1852-1866(2019)

DOI: $10.31801 /$ cfsuasmas.474512

ISSN 1303-5991 E-ISSN 2618-6470

http://communications.science.ankara.edu.tr/index.php?series $=\mathrm{A} 1$

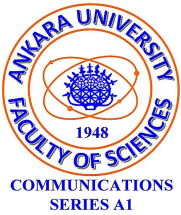

\title{
SCHATTEN-VON NEUMANN CHARACTERISTIC OF INFINITE TRIDIAGONAL BLOCK OPERATOR MATRICES
}

\author{
PEMBE IPEK AL AND ZAMEDDIN I. ISMAILOV
}

\begin{abstract}
In this paper, the boundedness and compactness properties of infinite tridiagonal block operator matrices in the direct sum of Hilbert spaces are studied. The necessary and sufficient conditions for these operators belong to Schatten-von Neumann class are given. Then, the results are supported by applications.
\end{abstract}

\section{INTRODUCTION}

The general theory of singular or characteristic numbers for linear compact operators in Hilbert spaces has been investigated by Gohberg and Krein 11], Pietsch [18, [19. Schmidt 20] and von Neumann, Schatten 24] have used these important results in the theory of non-selfadjoint integral operators.

Now give one definition.

Definition 1. Let $\mathcal{H}$ be a Hilbert space, $C_{\infty}(\mathcal{H})$ be a class of linear compact operators in $\mathcal{H}$, then $\left(A^{*} A\right)^{1 / 2} \in C_{\infty}(\mathcal{H})$. The eigenvalues of the operator $\left(A^{*} A\right)^{1 / 2}$ are called the s-numbers of the operator $A$. We shall enumerate the nonzero s-numbers in decreasing order, taking account of their multiplicities, so that

$$
s_{n}(A)=\lambda_{n}\left(\left(A^{*} A\right)^{1 / 2}\right), n=1,2, \ldots
$$

The Schatten-von Neumann operator ideals are defined as

$$
C_{p}(\mathcal{H})=\left\{A \in C_{\infty}(\mathcal{H}): \sum_{n=1}^{\infty} s_{n}^{p}(A)<\infty\right\}, 1 \leq p \leq \infty
$$

(see [1]).

Received by the editors: October 24, 2018; Accepted: February 12, 2019.

2010 Mathematics Subject Classification. 47A05, 47A10.

Key words and phrases. Direct sum of Hilbert spaces, infinite tridiagonal block operator matrices, compact operator, Schatten-von Neumann classes.

(C)2019 Ankara University Communications Faculty of Sciences University of Ankara-Series A1 Mathematics and Statistics 
The traditional tool of computational science is the theory of block matrices. There are many applications related to spectral problems of block operator matrices in 3 .

In [8], the traditional infinite direct sum of Hilbert spaces $H_{n}, n \geq 1$ is defined as

$$
H=\bigoplus_{n=1}^{\infty} H_{n}=\left\{u=\left(u_{n}\right): u_{n} \in H_{n}, n \geq 1, \sum_{n=1}^{\infty}\left\|u_{n}\right\|_{H_{n}}^{2}<+\infty\right\}
$$

Recall that $H$ is a Hilbert space with the norm induced by the inner product

$$
(u, v)_{H}=\sum_{n=1}^{\infty}\left(u_{n}, v_{n}\right)_{H_{n}}, u, v \in H
$$

Throughout this paper, we use the following notations:

$$
\begin{aligned}
& (\cdot, \cdot)_{H}:=(\cdot, \cdot),\|\cdot\|_{H}:=\|\cdot\| \text { and } \\
& (\cdot, \cdot)_{H_{n}}:=(\cdot, \cdot)_{n},\|\cdot\|_{H_{n}}:=\|\cdot\|_{n}, n \geq 1 .
\end{aligned}
$$

There are numerous physical problems arising in the modelling of processes of multiparticle quantum mechanics, quantum field theory and the physics of rigid bodies. These problems support to study the theory of linear operators in the direct sum of Hilbert spaces (see [14, [25] and references in them). In addition, they have been widely studied in view of spectral analysis of finite or infinite dimensional real and complex entries special matrices (upper and lower triangular double-band or third-band or Toeplitz types) in sequences spaces $\omega, c, c_{0}, b s, b \omega_{p}, l_{p}$ (see [1], [2], 4], [5], [6], [9], [23]).

Some spectral analysis of $2 \times 2$ and $3 \times 3$ types block operator matrices have been studied in [13, 15], 22]. The structure of spectrum of diagonal operator matrices has been obtained in [17. Furthermore, the compactness property and membership to Schatten-von Neumann classes of diagonal operator matrices in the direct sum of Hilbert spaces have been examined in [12].

In the present paper, we study the compactness properties of infinite tridiagonal block operator matrices in the infinite direct sum of Hilbert spaces. Then, membership to Schatten-von Neumann classes of these type operators is examined. Finally, some examples are provided as an application of our results.

Throughout this paper, sets of linear bounded operators, compact operators, Schatten-von Neumann classes from any Hilbert space $H_{1}$ to another Hilbert space $\mathrm{H}_{2}$ and singular numbers of any compact operator are denoted, respectively, by $L\left(H_{1}, H_{2}\right), C_{\infty}\left(H_{1}, H_{2}\right), C_{p}\left(H_{1}, H_{2}\right), 1 \leq p<\infty$ and $s_{n}(\cdot), n \geq 1$, respectively. If $H_{1}=H_{2}=H$, it is denoted by $L(H)=L(H, H), C_{\infty}(H)=C_{\infty}(H, H)$ and $C_{p}(H)=C_{p}(H, H)$. 


\section{BOUNDEDNESS AND COMPACTNESS OF INFINITE TRIDIAGONAL BLOCK OPERATOR MATRICES}

In this section, we will investigate the continuity and compactness property of tridiagonal block operator matrices which have the following form

$$
T=\left(\begin{array}{ccccccc}
A_{1} & B_{1} & & & & & \\
C_{1} & A_{2} & B_{2} & & & & \\
& C_{2} & A_{3} & B_{3} & & 0 & \\
& & \ddots & \ddots & \ddots & & \\
& 0 & & C_{n-1} & A_{n} & B_{n} & \\
& & & & \ddots & \ddots & \ddots
\end{array}\right)
$$

on the direct sum $H=\bigoplus_{n=1}^{\infty} H_{n}$ of Hilbert spaces $H_{n}$ in the case $A_{n} \in L\left(H_{n}\right)$, $B_{n} \in L\left(H_{n+1}, H_{n}\right)$ and $C_{n} \in L\left(H_{n}, H_{n+1}\right)$ for $n \geq 1$.

The operators $A, B, C: H \rightarrow H$ are defined as

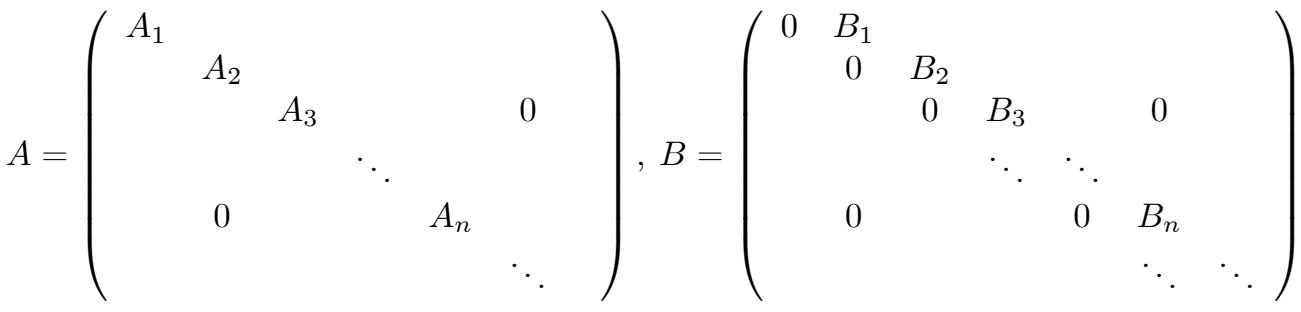

$$
\begin{aligned}
& C=\left(\begin{array}{cccccc}
0 & & & & & \\
C_{1} & 0 & & & & \\
& C_{2} & 0 & & & 0 \\
& & \ddots & \ddots & & \\
& 0 & & C_{n} & 0 & \\
& & & & \ddots & \ddots
\end{array}\right)
\end{aligned}
$$

First, let us prove the following theorem.

Theorem 2. $T \in L(H)$ if and only if

$$
\max \left\{\sup _{n \geq 1}\left\|A_{n}\right\|, \sup _{n \geq 1}\left\|B_{n}\right\|, \sup _{n \geq 1}\left\|C_{n}\right\|\right\}<\infty .
$$

In this case,

$$
\left(\|A\|^{2}+\|B\|^{2}+\|C\|^{2}\right)^{1 / 2} \leq\|T\| \leq\|A\|+\|B\|+\|C\| .
$$

Proof. Let $T \in L(H)$. Then, we have

$$
\|T x\| \leq\|T\|\|x\|
$$


for every $x \in H$. Later, in following calculations it will be assumed that $C_{0}=0$. One can easily calculate that it holds

$$
\left\|\left(0,0, \ldots, 0, C_{n-1} x_{n}, A_{n} x_{n}, B_{n} x_{n}, 0, \ldots\right)\right\| \leq\|T\|\left\|x_{n}\right\|_{n}
$$

for each special element $x=\left(x_{n}\right)$ in the form of

$$
x=\left(x_{n}\right)=\left(0,0, \ldots, 0, x_{n}, 0, \ldots\right) \in H, n \geq 1 .
$$

Thus, for any $n \geq 1$ we get

$$
\left\|C_{n-1} x_{n}\right\|_{n-1}^{2}+\left\|A_{n} x_{n}\right\|_{n}^{2}+\left\|B_{n} x_{n}\right\|_{n+1}^{2} \leq\|T\|^{2}\left\|x_{n}\right\|_{n}^{2} .
$$

We also have these inequalities

$$
\begin{aligned}
& \left\|C_{n-1} x_{n}\right\|_{n-1} \leq\|T\|\left\|x_{n}\right\|_{n}, \\
& \left\|A_{n} x_{n}\right\|_{n} \leq\|T\|\left\|x_{n}\right\|_{n} \text { and } \\
& \left\|B_{n} x_{n}\right\|_{n} \leq\|T\|\left\|x_{n}\right\|_{n}, \quad n \geq 1 .
\end{aligned}
$$

Hence,

$$
\left\|A_{n}\right\| \leq\|T\|,\left\|B_{n}\right\| \leq\|T\|,\left\|C_{n}\right\| \leq\|T\|, n \geq 1
$$

that is,

$$
\max \left\{\sup _{n \geq 1}\left\|A_{n}\right\|, \sup _{n \geq 1}\left\|B_{n}\right\|, \sup _{n \geq 1}\left\|C_{n}\right\|\right\} \leq\|T\|<\infty .
$$

Conversely, assume that

$$
\max \left\{\sup _{n \geq 1}\left\|A_{n}\right\|, \sup _{n \geq 1}\left\|B_{n}\right\|, \sup _{n \geq 1}\left\|C_{n}\right\|\right\}<\infty .
$$

In this case, for every $x=\left(x_{n}\right) \in H$ we have

$$
\begin{aligned}
\|B x\|^{2} & =\sum_{n=1}^{\infty}\left\|B_{n} x_{n+1}\right\|_{n}^{2} \\
& \leq \sum_{n=1}^{\infty}\left\|B_{n}\right\|^{2}\left\|x_{n+1}\right\|_{n+1}^{2} \\
& \leq\left(\sup _{n \geq 1}\left\|B_{n}\right\|\right)^{2}\|x\|^{2} .
\end{aligned}
$$

Therefore, we obtain

$$
\|B\| \leq \sup _{n \geq 1}\left\|B_{n}\right\| .
$$

Similarly, one can check that it holds

$$
\|C\| \leq \sup _{n \geq 1}\left\|C_{n}\right\| .
$$

It is also well-known from [16

$$
\|A\|=\sup _{n \geq 1}\left\|A_{n}\right\| .
$$


Consequently,

$$
\|T\| \leq\|A+B+C\| \leq\|A\|+\|B\|+\|C\| \leq \sup _{n \geq 1}\left\|A_{n}\right\|+\sup _{n \geq 1}\left\|B_{n}\right\|+\sup _{n \geq 1}\left\|C_{n}\right\| .
$$

Hence, $T \in L(H)$.

Assume that $T \in L(H)$. In this case, we have

$$
\|T x\| \leq\|T\|\|x\|
$$

for every $x \in H$.

Therefore, for each special elements in form

$$
x=\left(0,0, \ldots, 0, x_{n}, 0, \ldots\right) \in 0 \oplus 0 \oplus \ldots \oplus 0 \oplus H_{n} \oplus 0 \oplus \ldots, n \geq 1
$$

we have

$$
\left\|\left(0,0, \ldots, 0, C_{n-1} x_{n}, A_{n} x_{n}, B_{n} x_{n}, 0, \ldots\right)\right\| \leq\|T\|\|x\|_{n} .
$$

The last inequality implies that

$$
\left(\frac{\left\|C_{n-1} x_{n}\right\|_{n-1}^{2}}{\left\|x_{n}\right\|_{n}^{2}}+\frac{\left\|A_{n} x_{n}\right\|_{n}^{2}}{\left\|x_{n}\right\|_{n}^{2}}+\frac{\left\|B_{n} x_{n}\right\|_{n+1}^{2}}{\left\|x_{n}\right\|_{n}^{2}}\right)^{\frac{1}{2}} \leq\|T\|, \text { for } x_{n} \neq 0, n \geq 1 .
$$

Therefore, we get

$$
\left(\left(\sup _{n \geq 1}\left\|C_{n}\right\|\right)^{2}+\left(\sup _{n \geq 1}\left\|A_{n}\right\|\right)^{2}+\left(\sup _{n \geq 1}\left\|B_{n}\right\|\right)^{2}\right)^{1 / 2} \leq\|T\|,
$$

that is,

$$
\left(\|A\|^{2}+\|B\|^{2}+\|C\|^{2}\right)^{1 / 2} \leq\|T\|
$$

So, we have

$$
\left(\|A\|^{2}+\|B\|^{2}+\|C\|^{2}\right)^{1 / 2} \leq\|T\| \leq\|A\|+\|B\|+\|C\| .
$$

This completes the proof.

Remark 3. In the special and scalar case that

$$
\begin{aligned}
& A_{n}=v_{n}, C_{n}=-v_{n}, B_{n}=0, \\
& H_{n}=(\mathbb{R},|\cdot|), n \geq 1 \text { and } \\
& T: l_{1} \rightarrow l_{1}
\end{aligned}
$$

for the norm of corresponding infinite matrix with real entries, the following equality holds

$$
\|T\|=2 \sup _{k \geq 1}\left|v_{k}\right| .
$$

This result has been obtained in [2],21]. 
Remark 4. In the case of that

$$
\begin{aligned}
& A_{n}=a_{n}, n \geq 1, \\
& B_{n}=0, n \geq 1 \text { and } \\
& C_{n}=b_{n}, n \geq 1,
\end{aligned}
$$

some properties boundedness and norm of the corresponding lower triangle doubleband infinite matrix in $l_{p}, 1<p<\infty$ have been studied in [1].

Now, let us prove the following proposition on the compactness of the operator $T$ in $H$.

Theorem 5. $A, B, C \in C_{\infty}(H)$ if and only if $A_{n} \in C_{\infty}\left(H_{n}\right), B_{n} \in C_{\infty}\left(H_{n+1}, H_{n}\right)$, $C_{n} \in C_{\infty}\left(H_{n}, H_{n+1}\right)$ for any $n \geq 1$ and

$$
\begin{aligned}
& \lim _{n \rightarrow \infty}\left\|A_{n}\right\|=0, \\
& \lim _{n \rightarrow \infty}\left\|B_{n}\right\|=0, \\
& \lim _{n \rightarrow \infty}\left\|C_{n}\right\|=0,
\end{aligned}
$$

respectively.

Proof. The fact that $A \in C_{\infty}(H)$ if and only if for any $n \geq 1 A_{n} \in C_{\infty}\left(H_{n}\right)$ and $\lim _{n \rightarrow \infty}\left\|A_{n}\right\|=0$ has been proved in [12].

We prove this assertion for the operator $C$. It is clear that if $C \in C_{\infty}(H)$, then for any $n \geq 1 C_{n} \in C_{\infty}\left(H_{n}, H_{n+1}\right)$.

Let $\lim _{n \rightarrow \infty}\left\|C_{n}\right\|>0$. In that case, there exist $c>0$ and a sequence $\left(k_{n}\right) \subset \mathbb{N}$ such that

$$
\left\|C_{k_{n}}\right\|=\sup \left\{\frac{\left\|C_{k_{n}} u_{k_{n}}\right\|_{k_{n}+1}}{\left\|u_{k_{n}}\right\|_{k_{n}}}: u_{k_{n}} \in H_{k_{n}}, u_{k_{n}} \neq 0, n \geq 1\right\} \geq c>0 .
$$

In this case, we can also pick a sequence $\left(u_{k_{n}}^{*}\right) \subset H_{k_{n}}$ such that

$$
\frac{\left\|C_{k_{n}} u_{k_{n}}^{*}\right\|_{k_{n}+1}}{\left\|u_{k_{n}}^{*}\right\|_{k_{n}}} \geq c, n \geq 1 .
$$

If we define a subset of $H$ in the following form

$$
M:=\left\{(\underbrace{0,0, \ldots, 0}_{k_{n}-1}, \frac{u_{k_{n}}^{*}}{\left\|C_{k_{n}} u_{k_{n}}^{*}\right\|_{k_{n}+1}}, 0, \ldots) \in H: n \geq 1\right\},
$$

then we get

$$
\|u\| \leq \frac{1}{c}<\infty,
$$


for every $u \in M$, i.e., $M$ is a bounded set in $H$.

On the other hand, it is clear that

$$
C M=\left\{(\underbrace{0,0, \ldots, 0}_{k_{n}}, \frac{C_{k_{n}} u_{k_{n}}^{*}}{\left\|C_{k_{n}} u_{k_{n}}^{*}\right\|_{k_{n}+1}}, 0, \ldots) \in H: n \geq 1\right\} .
$$

Let show that the set $\overline{C M} \subset H$ is not compact. In order to see it, take an arbitrary sequence $\left(v_{n}\right) \subset \overline{C M}$. In this case, since $\left\|v_{n}\right\|_{H}=1, n \geq 1$, we obtain for the arbitrary $m, n \geq 1, m \neq n$

$\left\|v_{n}-v_{m}\right\|^{2}=\left(v_{n}-v_{m}, v_{n}-v_{m}\right)=\left\|v_{n}\right\|^{2}-\left(v_{n}, v_{m}\right)-\left(v_{m}, v_{n}\right)+\left\|v_{m}\right\|^{2}=1+1=2$.

Therefore, $\left(v_{n}\right) \subset \overline{C M}$ can not have a convergent subsequence.

Consequently,

$$
\varlimsup_{n \rightarrow \infty}\left\|C_{n}\right\|=0 .
$$

That is,

$$
\lim _{n}\left\|C_{n}\right\|=0 .
$$

On the contrary, in case when $C_{n} \in C_{\infty}\left(H_{n}, H_{n+1}\right), n \geq 1$ and $\lim _{n}\left\|C_{n}\right\|=0$ for the operators sequence

$$
K_{m}: H \rightarrow H,
$$

$$
K_{m}=\left(\begin{array}{cccccc}
0 & & & & & \\
C_{1} & 0 & & & & \\
& C_{2} & 0 & & & 0 \\
& & \ddots & \ddots & & \\
& 0 & & C_{m} & 0 & \\
& & & & \ddots & \ddots
\end{array}\right), m \geq 1 \text {, }
$$

we have

$$
\begin{aligned}
\left\|\left(C-K_{m}\right)(u)\right\|^{2} & =\sum_{n=m+1}^{\infty}\left(C_{n} u_{n}, C_{n} u_{n}\right)_{n+1} \\
& =\sum_{n=m+1}^{\infty}\left\|C_{n} u_{n}\right\|_{n+1}^{2} \\
& \leq \sum_{n=m+1}^{\infty}\left\|C_{n}\right\|^{2}\left\|u_{n}\right\|_{n}^{2} \\
& \leq \sup _{n \geq m+1}\left\|C_{n}\right\|^{2} \sum_{n=m+1}^{\infty}\left\|u_{n}\right\|_{n}^{2} \\
& \leq\left(\sup _{n \geq m+1}\left\|C_{n}\right\|^{2}\right)^{2}\|u\|^{2},
\end{aligned}
$$


for every $u \in H$.

Furthermore, since $\varlimsup_{n \rightarrow \infty}\left\|C_{n}\right\|=0$, it is clear that $\left(K_{m}\right)$ which is a sequence of operator in $B(H)$ converges to the operator $C$ in the operator norm. On the other hand, since $\left(K_{m}\right) \in C_{\infty}(H), m \geq 1$, then $C \in C_{\infty}(H)$ by the theorem of compact operators theory in 10 .

Similarly, it can be proved that $B \in C_{\infty}(H)$ if and only if $B_{n} \in C_{\infty}\left(H_{n+1}, H_{n}\right)$, $n \geq 1$ and

$$
\lim _{n \rightarrow \infty}\left\|B_{n}\right\|=0 .
$$

\section{Membership of $T$ to Schatten-von Neumann Classes}

Firstly, let us prove the following theorem for singular numbers of the operators $A, B, C$.

Theorem 6. Let $A, B, C \in C_{\infty}(H)$. Then, for the singular numbers of these operators the followings hold

$$
\begin{aligned}
& \left\{s_{m}(A): m \geq 1\right\}=\bigcup_{n=1}^{\infty}\left\{s_{k}\left(A_{n}\right): k \geq 1\right\}, \\
& \left\{s_{m}(B): m \geq 1\right\}=\bigcup_{n=1}^{\infty}\left\{s_{k}\left(B_{n}\right): k \geq 1\right\} \cup\{0\}, \\
& \left\{s_{m}(C): m \geq 1\right\}=\bigcup_{n=1}^{\infty}\left\{s_{k}\left(C_{n}\right): k \geq 1\right\} .
\end{aligned}
$$

Proof. For the diagonal block operator matrix

$$
A=\operatorname{diag}\left(A_{1}, A_{2}, \ldots, A_{n}, \ldots\right): H \rightarrow H,
$$

we get

$$
\sqrt{A^{*} A}=\operatorname{diag}\left(\sqrt{A_{1}^{*} A_{1}}, \sqrt{A_{2}^{*} A_{2}}, \ldots, \sqrt{A_{n}^{*} A_{n}}, \ldots\right) .
$$

From [12] we obtain

$$
\left\{s_{m}(A): m \geq 1\right\}=\bigcup_{n=1}^{\infty}\left\{s_{k}\left(A_{n}\right): k \geq 1\right\} .
$$

On the other hand, one can easily check that

$$
\sqrt{B^{*} B}=\operatorname{diag}\left(0, \sqrt{B_{1}^{*} B_{1}}, \sqrt{B_{2}^{*} B_{2}}, \ldots, \sqrt{B_{n-1}^{*} B_{n-1}}, \ldots\right): H \rightarrow H .
$$

From [12] we have

$$
\left\{s_{m}(B): m \geq 1\right\}=\bigcup_{n=1}^{\infty}\left\{s_{k}\left(B_{n}\right): k \geq 1\right\} \cup\{0\} .
$$


Similarly,

$$
\sqrt{C^{*} C}=\operatorname{diag}\left(\sqrt{C_{1}^{*} C_{1}}, \sqrt{C_{2}^{*} C_{2}}, \ldots, \sqrt{C_{n}^{*} C_{n}}, \ldots\right): H \rightarrow H .
$$

From 12 we get

$$
\left\{s_{m}(C): m \geq 1\right\}=\bigcup_{n=1}^{\infty}\left\{s_{k}\left(C_{n}\right): k \geq 1\right\}
$$

We can present the following results by using of Theorem 6 and 12 .

Corollary 7. If $A, B, C \in C_{p}(H), 1 \leq p \leq \infty$, then for every $n \geq 1$, $A_{n} \in C_{p}\left(H_{n}\right), B_{n} \in C_{p}\left(H_{n+1}, H_{n}\right)$ and $C_{n} \in C_{p}\left(H_{n}, H_{n+1}\right)$, respectively.

Proof. Since $A \in C_{p}(H), 1 \leq p<\infty$, the series $\sum_{m=1}^{\infty} s_{m}^{p}(A)$ is convergent. By the following inequality

$$
\sum_{k=1}^{\infty} s_{k}^{p}\left(A_{n}\right) \leq \sum_{m=1}^{\infty} s_{m}^{p}(A), n \geq 1
$$

one can see that the series $\sum_{k=1}^{\infty} s_{k}^{p}\left(A_{n}\right)$ is also convergent. Then, for every $n \geq 1, A_{n} \in C_{p}\left(H_{n}\right)$.

Similarly, we can prove that if $B, C \in C_{p}(H), 1 \leq p \leq \infty$, then for every $n \geq 1, B_{n} \in C_{p}\left(H_{n+1}, H_{n}\right), C_{n} \in C_{p}\left(H_{n}, H_{n+1}\right)$, respectively.

In the similar way, we can obtain the following corollary.

Corollary 8. $A, B, C \in C_{p}(H), 1 \leq p<\infty$ if and only if the series $\sum_{m=1}^{\infty} \sum_{n=1}^{\infty} s_{m}^{p}\left(A_{n}\right)$, $\sum_{m=1}^{\infty} \sum_{n=1}^{\infty} s_{m}^{p}\left(B_{n}\right)$ and $\sum_{m=1}^{\infty} \sum_{n=1}^{\infty} s_{m}^{p}\left(C_{n}\right)$ are convergent, respectively.

Note that in the case $B_{n}=C_{n}=0, n \geq 1$ the analogous problems have been investigated in Theorem 3.1, Theorem 3.2, Corollary 3.3 and Corollary 3.4 by [12]. Now, we can give the main result of this section.

Theorem 9. If $A \in C_{p}(H), B \in C_{q}(H)$ and $C \in C_{r}(H), 1 \leq p, q, r<\infty$, then $T \in C_{\alpha}(H), \alpha=\max (p, q, r)$. In this case,

$$
\sum_{n=1}^{\infty} s_{n}^{\alpha}(T) \leq 4^{\alpha} \sum_{n=1}^{\infty}\left(s_{n}^{\alpha}(A)+s_{n}^{\alpha}(B)+s_{n}^{\alpha}(C)\right) .
$$

Proof. First, assume that

$$
\sup _{n \geq 1}\left\|A_{n}\right\| \leq 1, \sup _{n \geq 1}\left\|B_{n}\right\| \leq 1, \sup _{n \geq 1}\left\|C_{n}\right\| \leq 1 .
$$


In this case, by a well-known property of singular numbers for every $n \geq 1$ we have

$$
\begin{aligned}
s_{3 n-2}^{\alpha}(T) & =s_{3 n-2}^{\alpha}(A+B+C) \\
& \leq\left(s_{2 n-1}(A+B)+s_{n}(C)\right)^{\alpha} \\
& \leq 2^{\alpha}\left(s_{2 n-1}^{\alpha}(A+B)+s_{n}^{\alpha}(C)\right) \\
& \leq 2^{\alpha}\left(\left(s_{n}(A)+s_{n}(B)\right)^{\alpha}+s_{n}^{\alpha}(C)\right) \\
& \leq 2^{\alpha}\left(2^{\alpha}\left(s_{n}^{\alpha}(A)+s_{n}^{\alpha}(B)\right)+s_{n}^{\alpha}(C)\right) \\
& =4^{\alpha} s_{n}^{\alpha}(A)+4^{\alpha} s_{n}^{\alpha}(B)+2^{\alpha} s_{n}^{\alpha}(C) \\
& \leq 4^{\alpha} s_{n}^{p}(A)+4^{\alpha} s_{n}^{q}(B)+2^{\alpha} s_{n}^{r}(C), \\
s_{3 n-1}^{\alpha}(T)= & s_{3 n-1}^{\alpha}(A+B+C) \\
\leq & \left(s_{2 n}(A+B)+s_{n}(C)\right)^{\alpha} \\
\leq & 2^{\alpha}\left(s_{2 n}^{\alpha}(A+B)+s_{n}^{\alpha}(C)\right) \\
\leq & 2^{\alpha}\left(s_{n+1}(A)+s_{n}(B)\right)^{\alpha}+2^{\alpha} s_{n}^{\alpha}(C) \\
\leq & 2^{\alpha}\left(2^{\alpha}\left(s_{n+1}^{\alpha}(A)+s_{n}^{\alpha}(B)\right)+2^{\alpha} s_{n}^{\alpha}(C)\right) \\
= & 4^{\alpha} s_{n+1}^{\alpha}(A)+4^{\alpha} s_{n}^{\alpha}(B)+2^{\alpha} s_{n}^{\alpha}(C) \\
\leq & 4^{\alpha} s_{n+1}^{p}(A)+4^{\alpha} s_{n}^{q}(B)+2^{\alpha} s_{n}^{r}(C)
\end{aligned}
$$

and

$$
\begin{aligned}
s_{3 n}^{\alpha}(T) & =s_{(3 n+1)-1}^{\alpha}(T) \\
& \leq\left(s_{2 n+1}(A+B)+s_{n}(C)\right)^{\alpha} \\
& \leq 2^{\alpha}\left(\left(s_{n+1}(A)+s_{n+1}(B)\right)^{\alpha}+s_{n}^{\alpha}(C)\right) \\
& \leq 4^{\alpha} s_{n+1}^{\alpha}(A)+4^{\alpha} s_{n+1}^{\alpha}(B)+2^{\alpha} s_{n}^{\alpha}(C) \\
& \leq 4^{\alpha} s_{n+1}^{p}(A)+4^{\alpha} s_{n+1}^{q}(B)+2^{\alpha} s_{n}^{r}(C) .
\end{aligned}
$$

From these inequalities and convergence of series $\sum_{n=1}^{\infty} s_{n}^{p}(A), \sum_{n=1}^{\infty} s_{n}^{q}(B), \sum_{n=1}^{\infty} s_{n}^{r}(C)$, we have that the series

$$
\sum_{n=1}^{\infty} s_{3 n-2}^{\alpha}(T), \sum_{n=1}^{\infty} s_{3 n-1}^{\alpha}(T) \text { and } \sum_{n=1}^{\infty} s_{3 n}^{\alpha}(T)
$$

are convergent. Consequently, the series $\sum_{n=1}^{\infty} s_{n}^{\alpha}(T)$ is convergent. This means that $T \in C_{\alpha}(H)$.

Now, consider the general case of operator $T$. In this case, for

$$
S=k T \text {, }
$$

where

$$
k=\frac{1}{1+c}, c=\max \left\{\sup _{n \geq 1}\left\|A_{n}\right\|, \sup _{n \geq 1}\left\|B_{n}\right\|, \sup _{n \geq 1}\left\|C_{n}\right\|\right\}
$$


one can obtain that

$$
\|S\|=k\|T\| \leq k \max \left\{\sup _{n \geq 1}\left\|A_{n}\right\|, \sup _{n \geq 1}\left\|B_{n}\right\|, \sup _{n \geq 1}\left\|C_{n}\right\|\right\}=\frac{c}{1+c} \leq 1 .
$$

According to above derived arguments

$$
\sum_{n=1}^{\infty} s_{n}^{\alpha}(S) \leq 4^{\alpha} \sum_{n=1}^{\infty}\left(s_{n}^{\alpha}(k A)+s_{n}^{\alpha}(k B)+s_{n}^{\alpha}(k C)\right) .
$$

That is,

$$
k^{\alpha} \sum_{n=1}^{\infty} s_{n}^{\alpha}(T) \leq 4^{\alpha} k^{\alpha} \sum_{n=1}^{\infty}\left(s_{n}^{\alpha}(A)+s_{n}^{\alpha}(B)+s_{n}^{\alpha}(C)\right) .
$$

Therefore,

$$
\sum_{n=1}^{\infty} s_{n}^{\alpha}(T) \leq 4^{\alpha} \sum_{n=1}^{\infty}\left(s_{n}^{\alpha}(A)+s_{n}^{\alpha}(B)+s_{n}^{\alpha}(C)\right) .
$$

Similarly, the following result can be proved.

Theorem 10. If for every $n \geq 1, A_{n}=A_{n}^{*}, C_{n}=B_{n}^{*}$ and $A \in C_{p}(H)$, $B+C \in C_{q}(H), 1 \leq p, q<\infty$, then

$$
\sum_{n=1}^{\infty}\left|\lambda_{n}(T)\right|^{\alpha} \leq 2^{\alpha} \sum_{n=1}^{\infty}\left(\left|\lambda_{n}(A)\right|^{\alpha}+\left|\lambda_{n}(B+C)\right|^{\alpha}\right),
$$

where $\alpha=\max (p, q)$.

Remark 11. In the case of that $A_{n}=B_{n}=0, n \geq 1, T \in C_{\infty}(H)$ (i.e. $\left.\lim _{n}\left\|C_{n}\right\|=0\right)$, the spectrum and the singular numbers of the operator $T$ are following forms

$$
\begin{aligned}
& \sigma(T)=\sigma_{r}(T)=\{0\}, \\
& \left\{s_{m}(T): m \geq 1\right\}=\bigcup_{n=1}^{\infty}\left\{s_{k}\left(C_{n}\right): k \geq 1\right\},
\end{aligned}
$$

respectively.

In this situation, the spectral radius $r_{\sigma}(T)$ satisfies the following important inequality

$$
r_{\sigma}(T)=0<\|T\|
$$

(see [7]). 
Remark 12. Using this method, the membership to Schatten-von Neumann classes of the following operator

$$
T=\left(\begin{array}{cccccc}
A_{11} & A_{12} & \ldots & A_{1 m} & & \\
A_{21} & A_{22} & \ldots & & A_{2 m+1} & \\
& & \ddots & & \ddots & \\
A_{k 1} & & & A_{k k} & & A_{k m+1} \\
& \ddots & & & \ddots & \ddots
\end{array}\right): H=\bigoplus_{n=1}^{\infty} H_{n} \rightarrow H
$$

can be studied.

\section{EXAMPLES}

In this section, we provide some examples as applications of our theorem.

Example 13. In the direct sum $H=\bigoplus_{n=1}^{\infty} H_{n}, H_{n}=(\mathbb{C},|\cdot|), n \geq 1$, consider the following tridiagonal infinite matrix with complex entries in form

$$
T=\left(\begin{array}{ccccccc}
a_{1} & b_{1} & & & & \\
c_{1} & a_{2} & b_{2} & & & \\
& c_{2} & a_{3} & b_{3} & & 0 & \\
& & \ddots & \ddots & \ddots & & \\
& 0 & & c_{n-1} & a_{n} & b_{n} & \\
& & & & \ddots & \ddots & \ddots
\end{array}\right): H \rightarrow H
$$

under the condition $\lim _{n \rightarrow \infty} a_{n}=\lim _{n \rightarrow \infty} b_{n}=\lim _{n \rightarrow \infty} c_{n}=0$. In this case, $T \in C_{\infty}(H)$ and if for every $n \geq 1$

$$
A_{n}:=a_{n}, B_{n}:=b_{n}, C_{n}:=c_{n},
$$

then

$$
\begin{aligned}
& s_{m}\left(A_{n}\right)=\left|\lambda_{m}\left(A_{n}\right)\right|=\left|a_{n}\right|, \\
& s_{m}\left(B_{n}\right)=\left|\lambda_{m}\left(B_{n}\right)\right|=\left|b_{n}\right| \text { and } \\
& s_{m}\left(C_{n}\right)=\left|\lambda_{m}\left(C_{n}\right)\right|=\left|c_{n}\right|, n, m \geq 1 .
\end{aligned}
$$

Hence, the singular number of operators $A, B, C$ are given in the following

$$
\begin{aligned}
& \left\{s_{m}(A): m \geq 1\right\}=\left\{\left|a_{n}\right|: n \geq 1\right\}, \\
& \left\{s_{m}(B): m \geq 1\right\}=\left\{\left|b_{n}\right|: n \geq 1\right\} \text { and } \\
& \left\{s_{m}(C): m \geq 1\right\}=\left\{\left|c_{n}\right|: n \geq 1\right\} .
\end{aligned}
$$

If

$$
\sum_{n=1}^{\infty}\left|a_{n}\right|^{p}<\infty, \sum_{n=1}^{\infty}\left|b_{n}\right|^{q}<\infty \text { and } \sum_{n=1}^{\infty}\left|c_{n}\right|^{r}<\infty, 1 \leq p, r, q<\infty
$$


then

$$
T \in C_{\alpha}\left(l_{2}\right), \alpha=\max (p, q, r) .
$$

Moreover, we get

$$
\sum_{n=1}^{\infty} s_{n}^{\alpha}(T) \leq 4^{\alpha} \sum_{n=1}^{\infty}\left(\left|a_{n}\right|^{\alpha}+\left|b_{n}\right|^{\alpha}+\left|c_{n}\right|^{\alpha}\right)
$$

Example 14. Let for $n \geq 1 H_{n}=\left(\mathbb{C}^{2},|\cdot|_{2}\right)$,

$$
A_{n}=\left(\begin{array}{cc}
0 & \alpha^{n} \\
\alpha^{n+1} & 0
\end{array}\right), B_{n}=\left(\begin{array}{cc}
0 & \beta^{n} \\
\beta^{n+1} & 0
\end{array}\right) \text { and } C_{n}=\left(\begin{array}{cc}
0 & \gamma^{n} \\
\gamma^{n+1} & 0
\end{array}\right)
$$

under the condition that $0 \leq|\alpha|,|\beta|,|\gamma|<1$.

Consider the following tridiagonal infinite block operator matrix with $2 \times 2$ order

$$
T=\left(\begin{array}{ccccccc}
A_{1} & B_{1} & & & & & \\
C_{1} & A_{2} & B_{2} & & & & \\
& C_{2} & A_{3} & B_{3} & & 0 & \\
& & \ddots & \ddots & \ddots & & \\
& 0 & & C_{n-1} & A_{n} & B_{n} & \\
& & & & \ddots & \ddots & \ddots
\end{array}\right): H \rightarrow H
$$

on the direct sum

$$
H=\bigoplus_{n=1}^{\infty} H_{n}
$$

Then $T \in C_{\infty}(H)$. Moreover, we have

$$
\left\|A_{n}\right\|=|\alpha|^{n},\left\|B_{n}\right\|=|\beta|^{n},\left\|C_{n}\right\|=|\gamma|^{n}, n \geq 1
$$

and

$$
\begin{aligned}
& \left\{s_{m}(A): m \geq 1\right\}=\left\{|\alpha|^{n},|\alpha|^{n+1}: n \geq 1\right\} \\
& \left\{s_{m}(B): m \geq 1\right\}=\left\{|\beta|^{n},|\beta|^{n+1}: n \geq 1\right\} \\
& \left\{s_{m}(C): m \geq 1\right\}=\left\{|\gamma|^{n},|\gamma|^{n+1}: n \geq 1\right\} .
\end{aligned}
$$

In this case, since $\sum_{m=1}^{\infty} s_{m}^{p}(A) \leq 2 \sum_{n=1}^{\infty}|\alpha|^{n p}<\infty, \sum_{m=1}^{\infty} s_{m}^{p}(B) \leq 2 \sum_{n=1}^{\infty}|\beta|^{n p}<\infty$ and $\sum_{m=1}^{\infty} s_{m}^{p}(C) \leq 2 \sum_{n=1}^{\infty}|\gamma|^{n p}<\infty, T \in C_{p}(H)$ and $\sum_{n=1}^{\infty} s_{n}^{p}(T) \leq 2 \sum_{n=1}^{\infty}\left(|\alpha|^{n}+|\beta|^{n}+|\gamma|^{n}\right)$, for any $1 \leq p<\infty$. 


\section{REFERENCES}

[1] Akhmedov, A. M. and El-Shabrawy, S. R., On the spectrum of the generalized lower triangle double-band matrices, International Conference on Functional Analysis Proceeding, 17-21 November 2010, Lviv, Ukraine, 2010, 12-13.

[2] Akhmedov, A. M. and El-Shabrawy, S. R., Notes on the spectrum of lower triangular doubleband matrices, Thai Journal of Mathematics 10, (2012), 415-421.

[3] Aurentz, J. L., and Trefethen, L. N., Block operators and spectral discretizations, SIAM Review 59, (2017), 423-446.

[4] Baliarsingh, P. and Dutta, S., On a spectral classification of the operator $\Delta_{v}^{r}$ over the sequence space $c_{0}$, Proceedings of the National Academy of Sciences, India Section A: Physical Sciences 84, 2014, 555-561.

[5] Böttcher, A. and Grudsky, S., Toeplitz Matrices, Asymptotic Linear Algebra and Functional Analysis, Berlin, Springer-Verlag, 1991.

[6] Böttcher, A. and Silbermann, B., Analysis of Teoplitz Operators, Berlin, Springer-Verlag, 1990.

[7] Demuth, M., Mathematical aspect of physics with non-selfadjoint operators, List of open problem, American Institute of Mathematics Workshop, 8-12 June 2015.

[8] Dunford, N. and Schwartz, J. T., Linear Operators I, New York, Interscience Publishers, 1958.

[9] El-Shabrawy, S. R., Spectra and fine spectra of certain lower triangular double-band matrices as operator on $c_{0}$, Journal of Inequalities and Applications 1, (2014), 2-9.

[10] Gohberg, I. C., Goldberg, S. and Kaashoek, M. A., Basic Classes of Linear Operators, Berlin, Birkhauser, 2003.

[11] Gohberg I. C., Krein M. G., Introduction to the Theory of Linear Non-Selfadjoint Operators in Hilbert Space, Rhode Island, USA, American Mathematical Society, 1969.

[12] Ismailov, Z. I., Otkun Çevik, E. and Unluyol, E., Compact inverses of multipoint normal differential operators for first order, Electronic Journal of Differential Equations 89, (2011), $1-11$.

[13] Jeribi A. Spectral Theory and Applications of Linear Operators and Block Operator Matrices, Switzerland, Springer, 2015.

[14] Kochubei, A. N., Symmetric operators and nonclassical spectral problems, Matematicheskie Zametki 25, (1979), 425-434.

[15] Nagel, R., The spectrum of unbounded operator matrices with non-diagonal domain, Journal of Functional Analysis 89, (1990), 291-302.

[16] Naimark, M. A. and Fomin, S. V., Continuous direct sums of Hilbert spaces and some of their applications, Uspekhi Mat Nauk 10, (1955), 111-142 (in Russian).

[17] Otkun Çevik, E. and Ismailov, Z. I., Spectrum of the direct sum of operators, Electronic Journal of Differential Equations 210, 2012, 1-8.

[18] Pietsch, A., Operators Ideals, North-Holland Publishing Company, Amsterdam, Holland, 1980.

[19] Pietsch, A., Eigenvalues and $s$-Numbers, Cambridge University Press, Londan, England, 1987.

[20] Schmidt. E., Zur theorie der linearen und nichtlinearen integralgleichungen Math. Ann. 64, (1907), 433-476.

[21] Srivastava, P. D. and Kumar, S., Fine spectrum of the generalized difference operator $\Delta_{v}$ on sequence space $l_{1}$, Thai Journal of Mathematics 8, (2010), 221-233.

[22] Tretter, Ch., Spectral Theory of Block Operator Matrices and Applications, Londan, Imperial CollegePress, 2008.

[23] Tripathy, B. C. and Das, R., Spectrum and fine spectrum of the lower triangular matrix $B(r, 0, s)$ over the sequences spaces, Appl Math Inf Sci 9, (2015), 2139-2145. 
[24] von Neumann, J. and Schatten, R., The cross-space of linear transformations, Ann. Math. 47, (1946), 608-630.

[25] Zettl, A. ,Sturm-Liouville Theory, USA, American Mathematical Society, 2005.

Current address: Pembe Ipek Al: Department of Mathematics, Faculty of Sciences, Karadeniz Technical University, Trabzon, Turkey

E-mail address: ipekpembe@gmail.com

ORCID Address: https://orcid.org/0000-0002-6111-1121

Current address: Zameddin I. Ismailov: Department of Mathematics, Faculty of Sciences, Karadeniz Technical University, Trabzon, Turkey

E-mail address: zameddin.ismailov@gmail.com

ORCID Address: http://orcid.org/0000-0001-5193-5349 